RECyT

Año 22 / № 33 / 2020 / 6-13

\title{
Evaluación de la variabilidad agromorfológica de poblaciones nativas de maíz de Misiones, Argentina
}

\section{Evaluation of the agromorphological variability of maize landraces in Misiones, Argentina}

\author{
Monica I. Heck ${ }^{1, *}$, Raquel A. Defacio ${ }^{2}$, Marcelo E. Ferrer ${ }^{2}$, Alfredo G. Cirilo², \\ Silvina I. Fariza' ${ }^{1}$, Adrián D. De Lucia ${ }^{1}$, Jorge A. Blaszchik ${ }^{1}$ \\ 1- Estación Experimental Agropecuaria Cerro Azul (CR Misiones) - INTA. Ruta Nacional 14, km 1085. Cerro Azul, Misiones. \\ 2- Estación Experimental Agropecuaria Pergamino (CR Buenos Aires Norte) - INTA. \\ Av. Pres. Arturo Frondizi Km 4,5. Pergamino, Buenos Aires. \\ *E-mail: heck.monica@inta.gob.ar
}

Recibido el 9 de noviembre de 2018, Aprobado el 20 de mayo de 2019.

\section{Resumen}

Las variedades locales de maíz presentan una gran relevancia socioeconómica en los sistemas agrícolas familiares de la provincia de Misiones. La pérdida de costumbres y tradiciones asociadas a su uso ponen en riesgo su conservación. Con el fin de aportar al conocimiento de la variabilidad genética local, contribuir a la conservación, revalorización y utilización de estos recursos, se estudiaron 16 poblaciones nativas de diferente origen y fecha de colecta mediante descriptores agromorfológicos. Los resultados permiten concluir que 1) las poblaciones nativas evaluadas albergan niveles considerables de variabilidad genética; 2) Se mantiene la identidad de razas dentro de cada origen; y 3) existe una tendencia al aumento de productividad en las colectas actuales. Esto pone de manifiesto, por un lado, la existencia de barreras geográficas y culturales que preservan el ideotipo, y por otro, la naturaleza dinámica de la conservación sometida a procesos de selección, sustitución y/o introgresión genética.

Palabras clave: Maíz; Recursos genéticos; Poblaciones nativas; Variabilidad genética; Conservación.

\section{Abstract}

Maize landraces present a great socio-economic relevance within the family farming systems in the province of Misiones. The losses of customs and traditions related to their use, endanger their conservation. In order to provide knowledge about the local genetic variability, conservation, valorization and utilization of these resources, 16 native populations, which belong to different origins and collection dates were studied by agromorphological descriptors. These findings allow us to conclude that (1) the evaluated native populations, hold significant levels of genetic variability, (2) the identity of races within each origin is maintained, and (3) there is a tendency to increase productivity in the current collections. On the one hand, these results highlight the existence of geographical and cultural barriers that preserve the ideotype and, on the other hand, the dynamic nature of conservation that has to undergo processes of selection, substitution and/or genetic introgression.

Keywords: Maize; Genetic resources; Native populations; Genetic variability; Conservation.

\section{Introducción}

El maíz (Zea mays, L.) presenta una gran diversidad genética, contenida en sus numerosas razas locales. La provincia de Misiones es el segundo centro de variabilidad genética del norte argentino con al menos 14 razas nativas de maíz identificadas [1, 2, 3, 4, 5]. Las mismas han sido mantenidas y mejoradas mediante prácticas agrícolas tradicionales por comunidades de la etnia Guaraní y pequeños agricultores de subsistencia. Presentan una gran adaptación al ambiente natural y antropológico en el que se desarrollaron [6, 7], su valor potencial reside en genes de resistencia a enfermeda- des y plagas, calidad nutritiva y adaptación a condiciones ambientales cambiantes [8,9]. Representan una fuente de características importantes para la adaptación local, la estabilidad económica y la sostenibilidad del agricultor [10].

Actualmente, el corrimiento de la frontera agrícola, los cambios en los sistemas productivos, la sustitución por cultivares comerciales y la pérdida de costumbres y tradiciones asociadas a su uso, representan una seria amenaza para la conservación de las variedades nativas de la región [11, 12, $13,3,4]$. Esto genera graves consecuencias sobre la producción agropecuaria y la seguridad alimentaria [14, 15, 13, 16]. Estudios previos realizados en razas locales de maíz proce- 
dentes de comunidades aborígenes de la etnia Guaraní del norte de la provincia de Misiones [17, 3, 4] y poblaciones nativas del noroeste argentino [2] reportan la presencia de altos niveles de diversidad genética en el germoplasma local. Teniendo en cuenta la importancia que revisten estos materiales en los sistemas productivos locales y al panorama global de erosión genética, la descripción de variedades nativas en las condiciones ambientales de la provincia es fundamental ya que permitirá detectar caracteres diferenciales que le otorguen valor agregado, beneficiando a los agricultores que las utilizan y conservan. En este contexto, el presente trabajo tiene como objetivo aportar al conocimiento del estado y evolución de la variabilidad genética de poblaciones nativas de maíz de la provincia de Misiones generando herramientas y capacidades de manejo para la conservación y aprovechamiento del germoplasma nativo.

\section{Materiales y Métodos}

\section{Material vegetal}

Se evaluaron 16 poblaciones nativas de la provincia de Misiones. La elección del material vegetal se realizó considerando diferentes orígenes, fechas de colecta y tipos raciales (Tabla 1). Los orígenes consistieron en dos sistemas culturales/productivos distintos: "Comunidades Mbya guaraní" y "Pequeños productores". Las colectas fueron realizadas en dos fechas distintas: año 1977 (Colecta antigua) y año 2008 (Colecta actual). Como testigos se utilizaron tres cultivares comerciales: Leales 25 plus, SH5090 y Centralmex. El último material corresponde a una variedad mejorada antigua de gran difusión pero que no se usa en la actualidad.

Tabla 1: Poblaciones nativas de maíz de la provincia de Misiones y testigos evaluados.

\begin{tabular}{|c|c|c|c|c|c|}
\hline $\mathbf{N}^{\circ}$ & Población & Forma racial & Origen & $\begin{array}{l}\text { Sitio de } \\
\text { colecta }\end{array}$ & $\begin{array}{l}\text { Fecha } \\
\text { colecta }\end{array}$ \\
\hline P1 & MNES01 & Catete oscuro & Mbya guaraní & El Soberbio & 2008 * \\
\hline P2 & MNESO2 & Avatí morotí ti & Mbya guaraní & El Soberbio & 2008 * \\
\hline P3 & MNESO3 & Avatí morotí mitá & Mbya guaraní & El Soberbio & 2008 * \\
\hline P5 & MNES05 & $\begin{array}{l}\text { D. amarillo } \\
\text { marlo fino }\end{array}$ & Productores & L.N.Alem & 2008 * \\
\hline P6 & MNESO6 & Dentado amarillo & Productores & $\begin{array}{c}\text { Picada } \\
\text { Propaganda }\end{array}$ & 2008 * \\
\hline P7 & MNES07 & Cravo & Productores & Montecarlo & 2008 * \\
\hline P8 & MNES08 & Semidentado & Productores & Puerto Rico & 2008 * \\
\hline P9 & MNES09 & Dentado amarillo & Productores & L.N.Alem & 2008 * \\
\hline P10 & MNES010 & Dentado blanco & Productores & L.N.Alem & 2008 * \\
\hline P11 & ARZM05-002 & Dentado amarillo & Productores & L. N. Alem & 1977 * * \\
\hline P12 & ARZM05-016 & Avatí morotí & Productores & Almafuerte & 1977 ** \\
\hline P13 & ARZM05-022 & Dentado blanco & Productores & Picada Yapeyú & 1977 ** \\
\hline P14 & ARZM05-026 & Cravo & Productores & Alberdi & 1977 ** \\
\hline P15 & ARZM05-030 & Tusón & Productores & San Ignacio & 1977 ** \\
\hline P16 & ARZM05-067 & Avatí Morotí Ti & Mbya guaraní & \begin{tabular}{c|} 
Campo Las \\
Monjas KM 300 \\
\end{tabular} & 1977 ** \\
\hline P17 & ARZM05-070 & Avatí Morotí Mitá & Mbya guaraní & $\begin{array}{c}\text { Campo LaS } \\
\text { Monjas KM } 301 \\
\end{array}$ & 1977 ** \\
\hline T18 & Leales 25 plus & Cristalino & Testigo & Comercial & actual \\
\hline $\mathrm{T} 19$ & Centralmex & Semidentado & Testigo & Comercial & antiguo \\
\hline $\mathrm{T} 20$ & SH5090 & Cristalino & Testigo & Comercial & actual \\
\hline
\end{tabular}

\section{Ambientes de evaluación y diseño experimental}

Los materiales se evaluaron en cuatro ambientes de la provincia (Tabla 2). Los mismos correspondieron a tres localidades (Aristóbulo del Valle, Santa Rita y Cerro Azul) y dos campañas agrícolas (2009/10 y 2010/11). El ambiente I correspondió a un suelo con valores característicos de suelos toscosos, con buena calidad química, contenidos adecuados de Materia Orgánica (MO), Nitrógeno (N), Fósforo (P) y pH, y altos de Potasio (K), Calcio (Ca) y Magnesio (Mg) (Tabla 2). El ambiente II fue un suelo rojo típico, de baja fertilidad, con valores limitantes para la mayoría de sus componentes ( $\mathrm{MO}, \mathrm{N}, \mathrm{P}, \mathrm{Ca}, \mathrm{Mg}$ ), $\mathrm{pH}$ bajo $\mathrm{y}$ alto tenor de aluminio (Al) (Tabla 2). Los ambientes III y IV correspondieron a suelos rojos-toscosos, con altos contenidos de bases ( $\mathrm{Ca}, \mathrm{K}$ y $\mathrm{Mg}$ ) y valores limitantes de MO, P y N. El ambiente IV correspondió a un suelo muy desgastado por la actividad agrícola, con contenidos altamente limitantes de $\mathrm{MO}$ y $\mathrm{N}$ y trazas de $\mathrm{Al}$ en el perfil del suelo (Tabla 2). Los ambientes I y II se sembraron el 3 y el 4 de noviembre de la campaña 2009/10, los ambientes III y IV se sembraron el 1 y el 2 de noviembre de la campaña 2010/11.

Tabla 2: Características químicas del suelo en los cuatro ambientes de evaluación.

\begin{tabular}{|c|c|c|c|c|}
\hline \multirow{2}{*}{ Característica } & \multicolumn{4}{|c|}{ Ambiente } \\
\cline { 2 - 5 } & $\begin{array}{c}\text { I } \\
\text { Aristóbulo } \\
\text { del Valle } \\
\mathbf{2 0 0 9 / 1 0}\end{array}$ & $\begin{array}{c}\text { II } \\
\text { Santa Rita } \\
\mathbf{2 0 0 9 / 1 0}\end{array}$ & $\begin{array}{c}\text { III } \\
\text { Cerro Azul } \\
\mathbf{2 0 1 0 / 1 1}\end{array}$ & $\begin{array}{c}\text { IV } \\
\text { Cerro Azul } \\
\mathbf{2 0 1 0 / 1 1}\end{array}$ \\
\hline $\mathrm{MO}(\%)$ & 3,70 & 2,22 & 2,43 & 1,49 \\
\hline $\mathrm{N}(\%)$ & 0,35 & 0,13 & 0,16 & 0,13 \\
\hline $\mathrm{P} 2 \mathrm{O5}$ (ppm) & 8,38 & 4,87 & 1,22 & 5,69 \\
\hline $\mathrm{K}$ (meq/100g) & 1,46 & 0,48 & 0,51 & 0,14 \\
\hline $\mathrm{Ca}$ (meq/100g) & 15,68 & 3,08 & 13,64 & 14,53 \\
\hline $\mathrm{Mg}$ (meq/100g) & 3,79 & 1,09 & 3,18 & 5,38 \\
\hline $\mathrm{pH}$ Agua & 6,45 & 5,02 & 5,84 & 5,21 \\
\hline $\mathrm{Relación} \mathrm{C/N}$ & 8,18 & 12,83 & 11,47 & 8,74 \\
\hline Al (meq/100g) & $\mathrm{nd}$ & 0,82 & $\mathrm{nd}$ & 0,38 \\
\hline
\end{tabular}

Para los experimentos se utilizó un diseño de bloques completos al azar (DBCA) con tres repeticiones. E1 tamaño de la parcela experimental fue de tres surcos de cinco metros de largo, distanciados $0,70 \mathrm{~m}$ entre sí. La densidad final fue de 57.200 plantas/hectárea (4 plantas/ metro lineal). La siembra y manejo del cultivo se hicieron conforme el manejo convencional de los productores de la región, es decir, labranza convencional, siembra manual y sin aplicación de fertilizantes. Se aplicaron herbicidas de preemergencia en la totalidad de los ensayos (3 1/ha de atrazina 50\%), volviéndose a aplicar $21 /$ ha de atrazina 50\% en estado vegetativo previo a V6 (sexta hoja totalmente desplegada). Además, se emplearon métodos manuales de remoción de malezas cuando se consideró necesario. 
Para el control de insectos del suelo y nematodos se utilizó Cipermetrina a razón de $200 \mathrm{~cm} 3 /$ ha en preemergencia.

\section{Variables fenotípicas analizadas}

En cada parcela experimental se registró la floración masculina (GDUM) y femenina (GDUF) en grados día, cuando el $50 \%$ de las plantas de la parcela experimental presentaron emisión de polen y estigmas visibles respectivamente. Los días transcurridos se transformaron a tiempo térmico (Unidades de grados días, GDU) según la fórmula de Andrade [18], asumiendo un valor de $8^{\circ} \mathrm{C}$ para la temperatura base [19]. Los datos termopluviométricos fueron suministrados por la Estación Meteorológica de INTA Cerro Azul. Se calculó la sincronía entre ambas floraciones (ASI, en grados días) y el largo total del ciclo (GDMF, en grados días). En el estadio R3 o grano lechoso [20] se marcaron 10 plantas con competencia completa, sobre las cuales se midió la altura de planta (ALTPL, en $\mathrm{cm}$ ) y mazorca (ALTMZ, en $\mathrm{cm}$ ), la relación entre ambas alturas (INS), el diámetro del tallo (DT, en mm), número total de hojas $(\mathrm{NH})$, área de la hoja bandera (AHB, en cm2) y de la espiga (AHE, en cm2). La cosecha se realizó luego de alcanzada la madurez fisiológica, con una humedad entre el 20 y $25 \%$. Al momento de la misma se determinó la prolificidad (PROL) y el número de plantas quebradas (PORQ, en \%) y volcadas (PORV, en \%). Sobre el surco central de cada unidad experimental, se registró el peso seco de la muestra ajustado al $15 \%$ de humedad y se calculó el rendimiento de grano por hectárea (REND, en kg ha-1) y el peso de mil granos (P1000, en g). Sobre 10 mazorcas de cada parcela se midió la longitud (LMZ, en $\mathrm{cm}$ ) y el diámetro (DMZ, en $\mathrm{mm}$ ), el número de hileras (NHIL) y de granos por hilera (GHIL) y la proporción de marlo (PMAR, en \%). De cada mazorca se extrajeron de la parte central 10 granos sobre los cuales se determinó el ancho (AGR, en mm), la longitud (LGR, en mm) y el grosor (GGR, en mm). Cada variable se midió de acuerdo a los protocolos propuestos en la lista de descriptores del IPGRI [21].

\section{Análisis estadístico}

Para representar la variabilidad existente en la colección de trabajo se calcularon los estadísticos de medida central y de dispersión más relevantes: media, valores mínimos, valores máximos y coeficiente de variación. La asociación entre caracteres agromorfológicos se realizó mediante un análisis de correlaciones de Pearson (r). Se utilizó un Análisis de Componentes Principales (ACP) y un análisis de clúster para clasificar las poblaciones y revelar la forma en que las variables individuales contribuyeron a la agrupación obtenida. Para ello se utilizaron las medias poblacionales de las 24 variables estudiadas y se estandarizó la matriz de datos con media igual a 0 y varianza igual a 1. Los Componentes Principales (CP) se calcularon a partir de la matriz de correlaciones entre los caracteres. Las variables con mayor carga $(>0,65)$ en cada $\mathrm{CP}$ se utilizaron para discriminar entre poblaciones [22]. La interpretación visual de los resultados se obtuvo mediante la construcción de un gráfico bidimensional, utilizando los dos primeros CP. Un Árbol de Mínima Distancia (MST) originado de la matriz de distancias Euclídeas fue superpuesto sobre el gráfico del ACP para facilitar la interpretación de los resultados. Para detectar el agrupamiento natural del germoplasma evaluado se utilizó el Análisis de Clúster, basado en la matriz de distancias Euclídeas estandarizadas y el método de encadenamiento UPGMA. La bondad de ajuste del dendrograma obtenido se determinó a través del coeficiente de correlación cofenética (CCC) [22]. Se realizó un análisis de varianza y un Test de DGC (nivel de significancia de $\mathrm{p}<0,05$ ) para probar diferencias significativas entre los grupos obtenidos. Todos los análisis fueron llevados a cabo usando el software estadístico Infostat Versión 2012 [23].

\section{Resultados}

\section{Variabilidad a nivel de caracteres}

Todos los caracteres presentaron un amplio rango de variación, en particular, asincronía floral, porcentaje de quebrado y volcado (Tabla 3). Caracteres como altura de inserción de mazorca, área de hoja bandera y de la espiga, peso de mil granos, proporción de marlo, prolificidad y rendimiento, presentaron valores máximos de 3 a 6 veces superiores a los mínimos. Esto pone en relieve la existencia de niveles considerables de variabilidad genética en el germoplasma nativo.

Tabla 3: Valores medios, coeficientes de variación y rangos obtenidos para poblaciones nativas de maíz de la provincia de Misiones.

\begin{tabular}{|l|l|l|l|l|}
\hline \multicolumn{1}{|c|}{ Variable } & \multicolumn{1}{|c|}{ Media } & \multicolumn{1}{c|}{ CV } & \multicolumn{1}{|c|}{ Rango } & Max/Min \\
\hline $\begin{array}{l}\text { Grados día a floración } \\
\text { femenina (GDUF) }\end{array}$ & 1192,0 & 13,12 & $768,5-1468,8$ & 1,91 \\
\hline $\begin{array}{l}\text { Grados día a floración } \\
\text { masculina (GDUM) }\end{array}$ & 1096,0 & 10,63 & $768,5-1326,1$ & 1,73 \\
\hline Asincronía floral (ASI) & 95,09 & 60,31 & $0-280$ & 280,0 \\
\hline $\begin{array}{l}\text { Grados día a madurez } \\
\text { fisiológica (GDMF) }\end{array}$ & 1507,0 & 8,98 & $1158-1705$ & 1,47 \\
\hline Altura de planta (ALTPL) & 206,25 & 18,56 & $118,3-303,5$ & 2,57 \\
\hline $\begin{array}{l}\text { Altura de inserción de } \\
\text { mazorca (ALTMZ) }\end{array}$ & 121,2 & 26,95 & $54,2-194$ & 3,58 \\
\hline $\begin{array}{l}\text { Altura de planta/ } \\
\text { Altura mazorca (INS) }\end{array}$ & 1,76 & 11,36 & $1,4-2,4$ & 1,71 \\
\hline Diámetro del tallo (DT) & 19,93 & 16,34 & $12,8-27,6$ & 2,16 \\
\hline Número total de hojas (NH) & 15,69 & 15,37 & $9,3-20,8$ & 2,24 \\
\hline Área de hoja bandera (AHB) & 135,01 & 30,04 & $55,4-285,4$ & 5,15 \\
\hline $\begin{array}{l}\text { Área de hoja de la } \\
\text { espiga (AHE) }\end{array}$ & 546,5 & 20,77 & $252,6-772,9$ & 3,06 \\
\hline Longitud de mazorca (LMZ) & 14,44 & 11,19 & $9,8-18,6$ & 1,90 \\
\hline
\end{tabular}




\begin{tabular}{|l|l|l|l|l|}
\hline Diámetro de mazorca (DMZ) & 41,24 & 13,19 & $28,3-52,6$ & 1,86 \\
\hline Número de hileras (NHIL) & 13,63 & 16,15 & $9,0-22,0$ & 2,44 \\
\hline $\begin{array}{l}\text { Número de granos } \\
\text { por hilera (GHIL) }\end{array}$ & 31,34 & 14,68 & $21,3-47$ & 2,21 \\
\hline Ancho de grano (AGR) & 8,55 & 6,88 & $6,8-9,95$ & 1,46 \\
\hline Longitud de grano (LGR) & 10,92 & 11,99 & $7,6-14,5$ & 1,91 \\
\hline Grosor de grano (GGR) & 4,13 & 9,16 & $3,2-5,2$ & 1,63 \\
\hline Peso de mil granos (P1000) & 185,5 & 19,76 & $107,1-329,7$ & 3,08 \\
\hline Rendimiento (REND) & 3236,5 & 36,62 & $1205-7902$ & 6,56 \\
\hline Proporción de marlo (PMAR) & 23,48 & 23,12 & $14,5-62,9$ & 4,34 \\
\hline $\begin{array}{l}\text { Porcentaje de } \\
\text { quebrado (PORQ) }\end{array}$ & 4,55 & 219,58 & $0-100$ & 100,0 \\
\hline Porcentaje de volcado (PORV) & 14,5 & 154,7 & $0-100$ & 100,0 \\
\hline Prolificidad (PROL) & 0,95 & 23,19 & $0,36-1,9$ & 5,28 \\
\hline
\end{tabular}

$\mathrm{CV}=$ coeficiente de variación. Max/Min= relación valor máximo y mínimo. Las unidades de medidas se detallan en Materiales y Métodos.

\section{Asociación de caracteres}

Las mayores correlaciones se dieron entre los caracteres fenológicos y morfológicos de planta (Tabla 4). En general, las poblaciones de ciclo largo presentaron mayor número de hojas, altura de planta y de inserción de mazorca y mayores períodos de tiempo para acumular biomasa. A nivel de caracteres de la mazorca se destacó la correlación entre el diámetro de la mazorca y el número de hileras. El rendimiento se asoció positivamente con el peso de mil granos, el diámetro de la mazorca, el número de hileras y de granos por hilera, y todos estos caracteres, se correlacionaron estrecha y positivamente con la biomasa total producida durante el ciclo.

Tabla 4: Coeficientes de correlación para variables agromorfológicas en poblaciones nativas de maiz de la provincia de Misiones.

\section{Clasificación de las poblaciones}

El análisis de clúster (Figura 1) permitió identificar siete grupos en el germoplasma nativo (valor de corte 5,21), mostrando un buen ajuste con el modelo $(\mathrm{CCC}=0,773)$. En el gráfico obtenido a partir del ACP (Figura 2), se pudo observar que los genotipos evaluados mantuvieron el patrón de agrupamiento obtenido con el análisis de conglomerados. De la variabilidad total observada, las dos primeras CP explicaron el 68,3\%. Las variables que más influyeron en la separación de las poblaciones fueron las relacionadas con el crecimiento vegetativo, las fenológicas y los componentes de rendimiento. Las poblaciones ubicadas en el lado positivo del CP1 tendieron a presentar ciclos más largos, mayor altura de planta y área foliar, mayores rendimientos y peso de mil granos. En tanto que los valores más altos del CP2 se asociaron con poblaciones de mazorcas largas, menor número de hileras y granos anchos (Figura 2).

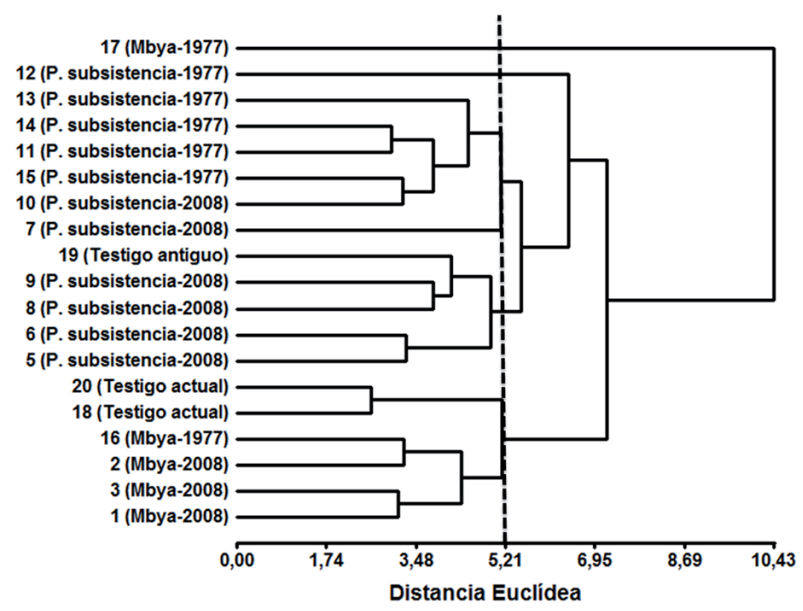

Figura 1: Análisis de clúster para variables agromorfológicas en poblaciones nativas de maíz de la provincia de Misiones, utilizando distancias Euclídeas y método de encadenamiento UPGMA.

GDUF GDUM ASI GDMF ALTPL ALTMZ INS DT NH LMZ DMZ NHIL GHIL AGR LGR GGR AHB AHE P1000 REND PMAR PORQ PORV

\begin{tabular}{|c|c|c|c|c|c|c|c|c|c|c|c|c|c|c|c|c|c|c|c|c|c|c|c|}
\hline GDUM & .99 & - & & & & & & & & & & & & & & & & & & & & & \\
\hline ASI & .93 & .86 & - & & & & & & & & & & & & & & & & & & & & \\
\hline GDMF & .90 & .91 & .78 & - & & & & & & & & & & & & & & & & & & & \\
\hline ALTPL & .88 & .87 & .82 & .78 & - & & & & & & & & & & & & & & & & & & \\
\hline ALTMZ & .91 & .90 & .82 & .85 & .98 & - & & & & & & & & & & & & & & & & & \\
\hline INS & -.90 & -.92 & -.78 & -.93 & -.83 & -.91 & - & & & & & & & & & & & & & & & & \\
\hline DT & .83 & .82 & .76 & .79 & .70 & .76 & -.81 & - & & & & & & & & & & & & & & & \\
\hline $\mathrm{NH}$ & .97 & .97 & .86 & .90 & .90 & .93 & -.92 & .85 & - & & & & & & & & & & & & & & \\
\hline LMZ & & & & & & & & & & - & & & & & & & & & & & & & \\
\hline DMZ & .51 & .54 & & .70 & .46 & .53 & -.62 & .52 & .55 & -.47 & - & & & & & & & & & & & & \\
\hline $\mathrm{NHIL}$ & & & & .43 & & & & & & -.61 & .80 & - & & & & & & & & & & & \\
\hline GHIL & .56 & .55 & .54 & .56 & .55 & .56 & -.52 & .49 & .51 & .40 & & & - & & & & & & & & & & \\
\hline AGR & & & & & & & & & & .49 & & -.54 & & - & & & & & & & & & \\
\hline LGR & .63 & .65 & .51 & .80 & .66 & .72 & -.73 & .63 & .67 & -.42 & .89 & .64 & .47 & & - & & & & & & & & \\
\hline GGR & -.59 & -.59 & -.53 & -.59 & -.62 & -.64 & .61 & -.52 & -.56 & & -.56 & & -.66 & & -.81 & - & & & & & & & \\
\hline $\mathrm{AHB}$ & .70 & .69 & .63 & .73 & .56 & .64 & -.74 & .67 & .66 & & .54 & & .67 & & .62 & -.63 & - & & & & & & \\
\hline AHE & .92 & .91 & .86 & .84 & .83 & .86 & -.87 & .83 & .93 & & .44 & & .51 & & .53 & -.45 & .78 & - & & & & & \\
\hline P1000 & .41 & .46 & & .61 & .55 & .57 & -.54 & .46 & .53 & & .60 & & & .62 & .66 & & & .47 & - & & & & \\
\hline REND & .53 & .56 & .41 & .76 & .50 & .58 & -.65 & .57 & .57 & & .85 & .54 & .59 & & .85 & -.58 & .65 & .50 & .72 & - & & & \\
\hline PMAR & & -.42 & & -.51 & & & .47 & & & & -.44 & & -.47 & & -.63 & .67 & & & & -.54 & - & & \\
\hline PORQ & -.50 & -.56 & & -.62 & & -.40 & & & -.47 & & -.61 & & -.42 & & -.60 & .56 & & & & -.62 & .71 & - & \\
\hline PORV & .20 & & .48 & & & & -.40 & .56 & .42 & & & & & & & & & & & & & & - \\
\hline PROL & -.46 & -.47 & & -.55 & -.58 & -.58 & .48 & -.40 & -.53 & & -.71 & -.49 & & & -.78 & .59 & -.44 & & -.58 & -.62 & & & \\
\hline
\end{tabular}

Nomenclatura de las variables como en Tabla 3. 
Los grupos reflejaron diferencias de rendimiento y sus componentes, longitud y sincronía del ciclo y desarrollo vegetativo (Figura 2, Tabla 5). El Grupo 1 estuvo constituido por una sola población harinosa procedente de comunidades Mbya (P17) colectada en 1977, con el ciclo más precoz y sincrónico, el menor porte y área foliar, los rendimientos más bajos, con mazorcas largas y finas, de bajo número de hileras y granos pequeños. El Grupo 2 comprendió cuatro poblaciones harinosas de la etnia guaraní (P1, P2, P3 y P16) de ciclo y porte intermedio, bajos rendimientos y sus componentes. El Grupo 3 comprendió los dos testigos cristalinos actuales (T18 y T20), de ciclo intermedio-corto y sincrónico, porte bajo, y valores intermedios-altos de rendimiento y sus componentes. El Grupo 4 formado en su mayoría por poblaciones de pequeños productores de subsistencia procedentes de la colecta antigua (P10, P11, P13, P14 y P15), con valores intermedios-altos de rendimiento y sus componentes, desarrollo vegetativo y ciclo. El Grupo 5 formado por una población de granos harinosos (P12) con valores intermedios-altos para la mayoría de los caracteres, diferenciado del grupo anterior por sus mazorcas largas y finas, de bajo número de hileras y granos anchos y profundos. El Grupo 6 formado por una población dentada perteneciente a la colecta actual (P7), de gran porte y área foliar, ciclo muy largo y asincrónico, altos rendimientos, mazorcas cortas de gran diámetro y número de hileras, con granos largos y finos. El Grupo 7, estuvo formado por el testigo antiguo (T19) y cuatro poblaciones dentadas y semidentadas pertenecientes a pequeños productores derivadas de la colecta actual (P5, P6, P8, P9). Este grupo comprendió poblaciones de gran porte, con altos rendimientos y peso de mil granos y ciclo muy largo y asincrónico, con mazorcas largas de gran diámetro con granos anchos y profundos.

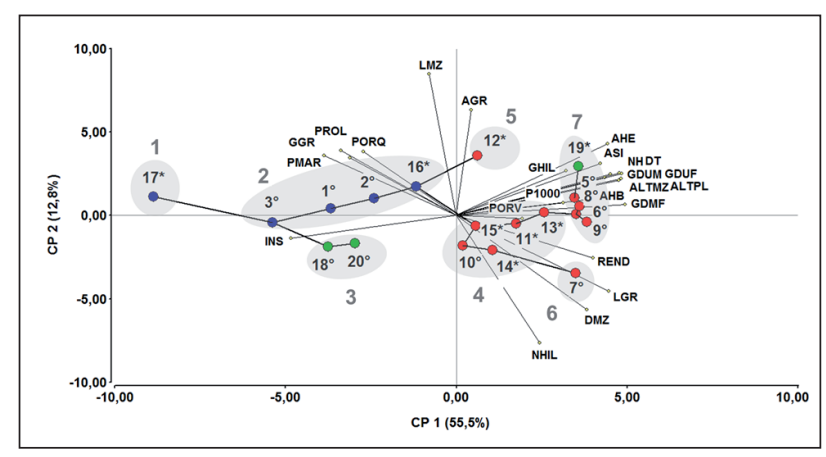

Figura 2: Dispersión de las poblaciones nativas en las primeras dos componentes principales. Se muestran los grupos identificados en el dendrograma y un Árbol de Mínima Distancia (MST). Referencias: Colecta antigua $\left({ }^{*}\right)$, Colecta actual $\left({ }^{\circ}\right)$, Mbya Guaraní (círculo azul), Testigos (círculo verde) y Pequeños productores (círculo rojo). Nomenclatura de las variables como en Tabla 3.
Tabla 5: Media de los caracteres agromorfológicos para los siete grupos resultantes del Análisis de Clúster.

\begin{tabular}{|c|c|c|c|c|c|c|c|}
\hline Variable & Grupo 1 & Grupo 2 & Grupo 3 & Grupo 4 & Grupo 5 & Grupo 6 & Grupo 7 \\
\hline GDUF & $907,5^{\mathrm{a}}$ & $1137,7^{c}$ & $1037,2^{b}$ & $1213,0^{c}$ & $1326,2^{d}$ & $1290,2^{\mathrm{d}}$ & $1286,8^{d}$ \\
\hline GDUM & $867,4^{a}$ & $1061,6^{b}$ & $1000,1^{b}$ & $1113,8^{c}$ & $1172,2^{c}$ & $1154,3^{c}$ & $1166,4^{c}$ \\
\hline ASI & $40,1^{\mathrm{a}}$ & $76,1^{\mathrm{b}}$ & $37,1^{\text {a }}$ & $99,2^{\mathrm{b}}$ & $154,1^{c}$ & $135,8^{c}$ & $120,4^{c}$ \\
\hline GDMF & $1290,4^{\mathrm{a}}$ & $1427,2^{b}$ & $1457,3^{b}$ & $1529,9^{b}$ & $1584,1^{\mathrm{b}}$ & $1557,0^{\mathrm{b}}$ & $1586,8^{b}$ \\
\hline ALTPL & $163,6^{\mathrm{a}}$ & $193,0^{b}$ & $164,4^{\mathrm{a}}$ & $207,8^{\mathrm{b}}$ & $208,4^{b}$ & $233,3^{c}$ & $234,8^{c}$ \\
\hline ALTMZ & $77,4^{\mathrm{a}}$ & $106,8^{b}$ & $87,3^{\mathrm{a}}$ & $124,8^{b}$ & $124,8^{b}$ & $142,5^{c}$ & $146,5^{c}$ \\
\hline INS & $2,20^{c}$ & $1,86^{\mathrm{b}}$ & $1,93^{b}$ & $1,71^{\mathrm{a}}$ & $1,68^{\mathrm{a}}$ & $1,70^{\mathrm{a}}$ & $1,64^{\mathrm{a}}$ \\
\hline $\mathrm{NH}$ & $13,55^{\mathrm{a}}$ & $16,27^{b}$ & $15,25^{b}$ & $17,75^{c}$ & $18,10^{c}$ & $18,40^{c}$ & $18,47^{c}$ \\
\hline AHB & $90,97^{a}$ & $112,70^{\mathrm{a}}$ & $107,90^{\mathrm{a}}$ & $140,88^{b}$ & $142,65^{b}$ & $155,27^{b}$ & $159,31^{\mathrm{b}}$ \\
\hline AHE & $381,4^{a}$ & $488,1^{\mathrm{a}}$ & $417,0^{\mathrm{a}}$ & $566,0^{\mathrm{b}}$ & $679,0^{\mathrm{b}}$ & $583,6^{\mathrm{b}}$ & $614,8^{b}$ \\
\hline LMZ & $15,58^{\mathrm{b}}$ & $14,41^{\mathrm{b}}$ & $14,66^{b}$ & $13,91^{b}$ & $15,80^{b}$ & $12,63^{\mathrm{a}}$ & $14,91^{\mathrm{b}}$ \\
\hline DMZ & $30,60^{a}$ & $34,84^{\mathrm{b}}$ & $43,98^{d}$ & $44,11^{\mathrm{d}}$ & $37,38^{c}$ & $48,50^{\mathrm{e}}$ & $43,75^{d}$ \\
\hline NHIL & $10,75^{a}$ & $11,67^{a}$ & $14,64^{c}$ & $14,69^{c}$ & $13,28^{b}$ & $19,38^{d}$ & $13,17^{b}$ \\
\hline GHIL & $29,35^{a}$ & $29,97^{\mathrm{a}}$ & $29,84^{a}$ & $30,50^{\mathrm{a}}$ & $30,90^{\mathrm{a}}$ & $31,43^{a}$ & $34,50^{b}$ \\
\hline AGR & $8,38^{b}$ & $8,54^{\mathrm{b}}$ & $8,80^{\mathrm{b}}$ & $8,40^{\mathrm{b}}$ & $8,78^{b}$ & $7,28^{a}$ & $8,91^{\mathrm{b}}$ \\
\hline LGR & $8,53^{\mathrm{a}}$ & $9,54^{\mathrm{b}}$ & $10,51^{c}$ & $11,48^{\mathrm{d}}$ & $9,58^{b}$ & $12,50^{\mathrm{e}}$ & $12,00^{\mathrm{e}}$ \\
\hline GGR & $4,73^{d}$ & $4,30^{c}$ & $4,48^{c}$ & $4,02^{b}$ & $4,60^{d}$ & $3,73^{\mathrm{a}}$ & $3,86^{\mathrm{a}}$ \\
\hline P1000 & $159,9^{\mathrm{a}}$ & $163,1^{\mathrm{a}}$ & $184,1^{\mathrm{a}}$ & $189,4^{\mathrm{a}}$ & $171,2^{\mathrm{a}}$ & $162,8^{\mathrm{a}}$ & $212,2^{b}$ \\
\hline REND & $2125,0^{\mathrm{a}}$ & $2331,7^{\mathrm{a}}$ & $3577,2^{b}$ & $3326,6^{b}$ & $2994,0^{b}$ & $3652,5^{b}$ & $3919,0^{\mathrm{b}}$ \\
\hline PMAR & $29,15^{b}$ & $23,98^{\mathrm{a}}$ & $22,79^{a}$ & $23,07^{\mathrm{a}}$ & $26,1^{\mathrm{a}}$ & $23,2^{\mathrm{a}}$ & $22,24^{a}$ \\
\hline PORQ & $14,15^{b}$ & $6,21^{\mathrm{a}}$ & $2,35^{\mathrm{a}}$ & $3,95^{\mathrm{a}}$ & $5,83^{\mathrm{a}}$ & $2,43^{a}$ & $3,00^{\mathrm{a}}$ \\
\hline
\end{tabular}

Para cada grupo, letras diferentes indican diferencias estadísticas significativas $(\mathrm{p}<0,05)$ con el Test de DGC. Nomenclatura de las variables como en Tabla 3.

La distribución de las poblaciones en cada cuadrante del ACP mostró una clara separación de los materiales por tipo de endosperma, origen (Mbya Guaraní vs Pequeños productores de subsistencia) y fecha de colecta (1977 vs 2008) (Figura 1). En el lado positivo de la CP1 se ubicaron variedades dentadas y semidentadas (Grupo 4, 6 y 7) y harinosas (Grupo 5) procedentes de pequeños productores de subsistencia. En los cuadrantes superior e inferior izquierdo se localizaron poblaciones harinosas originarias de comunidades Mbya guaraní (Grupos 1 y 2) y testigos comerciales de tipo cristalino (Grupo3), respectivamente. Pudo observarse además, una relación entre la magnitud de los caracteres evaluados y la fecha de colecta: las poblaciones procedentes de pequeños agricultores de subsistencia colectadas por el BAG en 1977 (P11, P12, P13, P14, P15) mostraron valores medios para las variables evaluadas, en tanto que, la mayoría de las colectadas actualmente (P5, P6, P7, P8, P9, P19), resultaron más altas, con ciclos más largos y asincrónicos y rendimientos superiores. 


\section{Discusión}

La variabilidad genética observada a nivel de caracteres agromorfológicos en el germoplasma nativo es de gran utilidad para el mejoramiento genético y la conservación de los recursos genéticos. La generación y conservación de la variabilidad en poblaciones nativas está asociada a la multiplicidad de ambientes y técnicas de producción, el aislamiento geográfico, la recombinación entre poblaciones aledañas, el flujo de semillas entre localidades y la selección realizada por los agricultores para caracteres asociados al rendimiento o usos especifícos $[24,25,26,27]$.

El empleo de técnicas multivariadas permitió la identificación de poblaciones o grupos de poblaciones con características diferenciales para los caracteres evaluados, sentando las bases para futuros programas de mejoramiento locales. Los siete grupos obtenidos reflejan diferencias de rendimiento y sus componentes, crecimiento vegetativo, longitud del ciclo y sincronía floral. La disposición de los mismos en el gráfico biplot refleja una progresión gradual en el nivel de expresión de estos atributos a nivel de grupos, desde el más precoz, con plantas pequeñas, de bajos rendimientos (Grupo 1), hasta los más tardíos, con plantas de mayor porte y rendimiento (Grupos 6 y 7), pasando por los de expresión intermedia (Grupos 2, 3, 4 y 5). Este patrón de distribución de la diversidad es un reflejo de la biología reproductiva de la especie y el largo proceso co-evolutivo de agricultores y sus cultivos [28], sometido a procesos de migración y deriva, selección natural y artificial, extinción local y recolonización [24, 25, 4]. Cada año los agricultores toman decisiones que afectan la diversidad genética de sus cultivos y con el paso del tiempo provocan cambios en la estructura genética de sus poblaciones, al seleccionar plantas con características deseables, al definir el tamaño de la población a sembrar, la cantidad de semilla introducida y conservada y/o al escoger una práctica de manejo específica $[24,25]$.

La diferenciación fenotípica observada en las poblaciones procedentes de la colecta actual (Grupo 6 y 7) con respecto a las colectas más antiguas (Grupo 4), la tendencia a mayores rendimientos, crecimiento vegetativo y longitud del ciclo en las colectas actuales y la asociación del testigo antiguo (T19) con las poblaciones de tipo dentado y semidentado de la colecta actual, sugieren cierto grado de erosión genética de estos materiales, asociada a procesos de selección, sustitución y/o "contaminación" con polen de variedades/ híbridos modernos. En general, las poblaciones nativas de maíz pasan de un agricultor a otro durante generaciones, sometidas a procesos de selección fenotípica directa o indirecta basada en caracteres fácilmente discernibles (fenológicos, vegetativos y de mazorca) [26]. La avidez del agricultor por probar nuevos materiales ha llevado, en muchos casos, al reemplazo de poblaciones nativas por variedades mejoradas más productivas, y en otros, a la recombinación consciente y/o inconsciente de ambos tipos de poblaciones [29, 25]. La cercanía geográfica con áreas de producción de Brasil y los estrechos vínculos de tipo familiar y social existente entre ambos países han llevado a una continua introducción de numerosos híbridos o variedades mejoradas provenientes de las zonas productivas limítrofes. Esta tendencia, sumada al manejo tradicional practicado en chacras de escasas superficies ( 25 ha. promedio), conduce a la siembra en áreas contiguas de materiales comerciales y poblaciones locales, sin barreras físicas ni temporales que permitan el aislamiento reproductivo. De esta manera, la "contaminación genética" causada por la dispersión de polen procedente de materiales comerciales ha ocurrido y seguirá ocurriendo. Una situación similar puede observarse en el contexto productivo de los pueblos originarios, donde, la continua expansión y transformación de la frontera agrícola comercial ha ocasionado importantes cambios estructurales y funcionales en los sistemas productivos. Los que antes disponían de vastas superficies, ricas en diversidad, aisladas cultural y productivamente, hoy se ven expuestos a superficies cada vez más reducidas, menos diversas y más susceptibles a la influencia de las costumbres y cultivos foráneos. La adopción de nuevas prácticas, como la cría de aves, conduce a la incorporación al sistema de nuevas variedades/ híbridos de otros tipos raciales, más adecuados para la alimentación animal. Esto, sumado a la cercanía de sus lotes de producción a los de otros agricultores locales, sin barreras de aislamiento espacial o temporal adecuadas, aumentaría el flujo génico entre ellas, provocando cierto grado de contaminación genética de las razas nativas, contribuyendo a explicar la gran diferenciación fenotípica observada al comparar algunas poblaciones de la colecta antigua y la actual (ej. P17 vs P3).

En contraparte, la diferenciación agromorfológica observada entre poblaciones nativas de maíz procedentes de "comunidades de la etnia guaraní" y aquellas pertenecientes a "pequeños productores de subsistencia" sugiere que los agricultores mantienen en cierto grado el ideotipo de mazorca de sus variedades y cualquier característica genéticamente vinculada, a través de factores de tipo racial y uso específicos, al tiempo que permiten que otras características evolucionen genéticamente. De esta manera al seleccionar plantas fuera de tipo mantienen el polimorfismo fenotípico entre variedades plantadas en áreas adyacentes, explicando la coexistencia de múltiples variedades a pesar de los numerosos factores que favorecen el intercambio genético entre ellas. Esto se corresponde con lo descripto por Louette y Smale [26] y Pressoir y Berthaud, [24] en sistemas productivos de México.

Estos resultados tienen profundas implicancias sobre la conservación de los recursos genéticos locales. Por un lado, realzan la importancia de la complementación de métodos de conservación de recursos genéticos en el agroecosistema donde se desarrollaron (in situ) con la realizada en bancos de germoplasma (ex situ). En la primera, la influencia de 
factores genéticos, ambientales y antrópicos genera "nueva variabilidad", manteniéndose intacto el contexto técnico, social, cultural y ambiental en el que estas poblaciones se desarrollaron [27], en tanto que en la segunda se resguarda las variedades originales y variantes útiles para mejoradores y/o agricultores [30]. La complementación de ambas estrategias de conservación permitirá restaurar el patrimonio genético original en sistemas agrícolas dañados, atenuar los riesgos provocados por la erosión genética y resguardar nuevas variantes útiles [31].

\section{Conclusiones}

Las poblaciones nativas de maíz de Misiones albergan niveles considerables de variabilidad genética para los caracteres agromorfológicos evaluados.

La diferenciación fenotípica observada entre poblaciones está fuertemente asociada con el tipo de endosperma, la arquitectura de planta, la duración del ciclo y los componentes de rendimiento.

La variabilidad mostró patrones consistentes con el origen y tiempo de colecta de los materiales, poniendo de manifiesto por un lado, a la existencia de barreras geográficas y culturales que preservan la identidad de razas dentro de cada origen y por otro, a la naturaleza dinámica de la conservación sometida a procesos de selección, sustitución y/o introgresión genética de materiales mejorados. Estos hallazgos tienen profundas implicancias sobre la conservación de los recursos genéticos locales, realzando la importancia de la complementación de métodos de conservación de recursos genéticos en el agroecosistema donde se desarrollaron (in situ) con la realizada en bancos de germoplasma (ex situ).

\section{Agradecimientos}

A los productores, comunidades Mbya y al Banco de Germoplasma de Maíz (INTA EEA Pergamino) por cedernos para su estudio muestras de las poblaciones nativas de maíz evaluadas. Este trabajo fue financiado por el Instituto Nacional de Tecnología Agropecuaria (INTA) y forma parte de la Tesis de Maestría de la MSc. Mónica Heck.

\section{Referencias}

1. Cámara Hernández, J. y Miante Alzogaray, A.M. Caracterización y clasificación en razas, de maíces nativos de la Provincia de Misiones, Argentina. In Proceedings of the IV Simposio de recursos genéticos para América Latina y el Caribe, Mar del Plata, Argentina. 2003.

2. Melchiorre, P.; Bartoloni, N.; Cámara Hernández, J. Phenetic relationships among native races of maize (Zea mays ssp. mays) from North-eastern Argentina (Misiones). Journal of Genetics and Breeding, 60:173-182. 2006.

3. Bracco, M.; Lia, V.V.; Hernández, J.C.; Poggio, L.; Gottlieb, A.M.
Genetic diversity of maize landraces from lowland and highland agro-ecosystems of Southern South America: implications for the conservation of native resources. Annals of Applied Biology, 160:308-321. 2012.

4. Bracco, M.; Lía, V.V.; Poggio, L.; Cámara Hernández, J.A.; Gottlieb, A.M. Caracterización genética de razas de maíz autóctonas de Misiones, Argentina. Revista Ciencia y Tecnología, 20:52-60. 2013.

5. Cámara Hernández, J.; Miante Alzogaray, A.M; Bellón, R.; Galmarini, A.J. Razas de maíz nativas de la Argentina. Ed. Fac. Agronomía, UBA, vol 1, 174 pp. 2012.

6. Esquinas Alcazar, J. Recursos genéticos vegetales: Bases para la seguridad alimentaria. Ceres, 118 (20):39-45. 1987.

7. Maxted, N.; Ford-Lloyd, B.; Hawkes, J.G. Complementary conservation strategies. In: Maxted N., Ford-Lloyd B., Hawkes J.C. (Eds.). Plant Genetic Conservation: The in situ Approach. Chapman \& Hall. London. pp.15-40. 1997.

8. Ruiz de Galarreta Gómez, J. I. Agrupación de poblaciones locales de maiz (Zea mays L.) mediante caracteres morfológicos y parámetros ambientales. Tesis doctoral. Universidad de Lleida. Salamanca. 161 pp.1993.

9. Araujo, P. M. y Nass, L. L. Caracterização e avaliação de populações de milho crioulo. Scientia Agricola, 59(3):589-593. 2002.

10. Mwololo, B.M. The role of farmers in biodiversity conservation of maize landraces through farming systems in Kenya. Journal of developments in sustainable agriculture, 5:155-177. 2010.

11. Bravo, A. L. El maíz en Ecuador. En: Maíz sustento y culturas en América Latina. Los impactos negativos de la globalización. Redes Amigos de la Tierra Uruguay \& Biodiversidad Sustento y Culturas. pp. 37-60. 2004.

12. Cámara Hernández, J. y Arancibia de Cabezas, D. Maíces andinos y sus usos en la quebrada de Humahuaca y regiones vecinas. Editorial Facultad de Agronomía. Universidad de Buenos Aires. 60 pp. 2007.

13. Berretta, A.; Albín, A.; Díaz, R.; Gómez, P. Recursos Fitogenéticos: Desafios y oportunidades. En: Estrategia en los recursos fitogenéticos para los países del Cono Sur. PROCISUR, IICA. Montevideo.172 pp. 2010.

14. Rao, R.V. y Hodgkin, T. Genetic diversity and conservation and utilization of plant genetic resources. Plant Cell, Tissue and Organ Culture, 68:1-19. 2002.

15. Brown, A.H.D. Indicators of genetic diversity, genetic erosion and genetic vulnerability for plant genetic resources for food and agriculture. FAO. 26 pp. 2008.

16. Govindaraj, M.; Vetriventhan, M.; Srinivasan, M. Importance of genetic diversity assessment in crop plants and its recent advances: an overview of its analytical perspectives. Genetics Research International, Volume 2015, Article ID 431487.14 pp. 2015.

17. Bracco, M. Caracterización genética del germoplasma de razas de maíz autóctonas provenientes del noreste 
argentino. Tesis Doctoral. Facultad de Ciencias Exactas y Naturales. Universidad de Buenos Aires. 140 pp. 2012.

18. Andrade, F. H.; Cirilo, A.G.; Uhart, S.A.; Otegui, M.E. Ecofisiología del cultivo de maíz. Editorial La Barrosa. Balcarce. Buenos Aires. 292 pp. 1996.

19. Cárcova, J.; Borrás, L.; Otegui, M. E. Ciclo ontogénico, dinámica del desarrollo y generación del rendimiento y la calidad en maíz. Capítulo 8. In: Producción de Granos. Bases funcionales para su manejo. Satorre et al. (Eds), Edit. Facultad de Agronomía-UBA. pp. 132-163. 2003.

20. Ritchie, S. W.; Hanway, J. J.; Benson, G. O. How a corn plant develops. Iowa State University. Special Report no. 48, Ames, IA, USA. 1993.

21. сімmYt/IBPGRI. Descriptores de maiz. México-Roma. 88 pp. 1991.

22. Sneath, P.H.A. y Sokal, R.R. Numerical Taxonomy: The principles and practice of numerical classification. In: Freeman, W.H., \& Co (eds.). San Francisco. 1973.

23. Di Rienzo, J.A.; Casanoves, F.; Balzarini, M.G.; Gonzalez, L.; Tablada M.; Robledo C.w. InfoStat versión 2012. InfoStat Group, Facultad de Ciencias Agropecuarias, Universidad Nacional de Córdoba, Argentina. http://www. infostat.com.ar. 2012.

24. Pressoir, G. y Berthaud, J. Population structure and strong divergent selection shape phenotypic diversification in maize landraces. Heredity, 92:95-101. 2004.

25. Jarvis, D. y Hodgkin, T. Farmer decision making and genetic diversity: linking multidisciplinary research to implementation on-farm. In Genes in the Field: On-Farm Conservation of Crop Diversity (SB Brush, ed). Lewis Publishers, Boca Raton, FL, USA. pp. 261-278. 2006.
26. Louette, D. y Smale, M. Farmer's seed selection practices and traditional maize varieties in Cuzalapa, Mexico. Euphytica, 113:25-41. 2000.

27. Lucchin, M.; Barcaccia, G.; Parrini, P. Characterization of a flint maize (Zea mays L. convar. Mays) Italian landrace: I. Morpho-phenological and agronomic traits. Genetic Resource and Crop Evolution, 50:315-327. 2003.

28. Hortelano Santa Rosa, R.; Gil Muñoz, A.; Santacruz Varela, A.; López Sánchez, H.; López, P.A.; Colín, S.M. Phenotypic diversity of maize landraces from the Central-Eastern Highland plateau of Puebla State, México. Fitotecnia Mexicana, 35(2):97-109. 2012.

29. Bellon, M.R.; Berthaud, J.; Smale, M.; Aguirre, J.A.; Taba, S.; Aragon, F.; Diaz, J.; Castro, H. Participatory landrace selection for on-farm conservation: An example from the Central Valleys of Oaxaca, Mexico. Genetic Resources and Crop Evolution, 50:401-416. 2003.

30. Seguel Benítez, I. Conservación de los recursos fitogenéticos ex-situ. En: Estrategia en recursos fitogenéticos para los países del Cono Sur”. PROCISUR. Montevideo. 144 pp. 2001.

31. Fernández Granda, L.; Crossa, J.; Fundora-Mayor, Z.; Gálvez Rodríguez, G.; Acuña Fernández, G.; Guevara Vázquez, C. Presencia de la variabilidad Ex Situ e In Situ en el germoplasma cubano de maíz (Zea mays L.). Importancia de la complementación de ambos enfoques de conservación. Cultivos Tropicales, 32 (4):21-34. 2011. 